\title{
REVISTA ESTOMATOLOGÍA
}

\author{
ESCUELA DE ODONTOLOGÍA \\ FACULTAD DE SALUD, UNIVERSIDAD DEL VALLE \\ Volumen $15 \mathrm{~N}^{\circ} 2,2007$ \\ Contenido
}

3 EDITORIAL

FREDDY MORENO

ARTIFICES Y Visionarios

4 ¿ES BÚHO O ES ÁGUILA?

FREDdY MORENo

Articulos Originales

6 FACTORES RELACIONADOS CON GINGIVITIS EN NIÑOS DE DOS INSTITUCIONES EDUCATIVAS DE CALI FACTORS RELATED WITH GINGIVITIS IN CHILDREN OF TWO EDUCATIONAL INSTITUTIONS FROM CALI

ingrid C. Solarte, Claudia L. Castillo, Karol L. Borrero, Diana C. Moscoso, Martha M. Aristizabal, Andrés F.

AguirRe

10 MORFOLOGÍA DENTAL EN DENTICIÓN MIXTA: CORRELACIÓN DE TRES RASGOS MORFOLÓGICOS DE DIENTES TEMPORALES Y PERMANENTES EN ESCOLARES DE CALI, COLOMBIA

MIXED DENTITION MORPHOLOGY CHARACTERISTICS: A CORRELATION BETWEEN 3 CROWN DENTAL MORPHOLOGY TRAITS IN TEMPORAL AND PERMANENT DENTITION IN SCHOLARS FROM CALI - COLOMBIA

luisa Aguirre, Diana Castillo, Diana Solarte, Freddy Moreno

Reporte de Caso

20 DENTINOGÉNESIS IMPERFECTA: REPORTE DE UN CASO

IMPERFECTA DENTINOGENESIS: A CASE REPORT

Patricia Trejos, Vanessa Hernando, Claudia S. De León

Articulos de ReVision

29 DIENTES SUPERNUMERARIOS. REVISIÓN DE LA LITERATURA

SUPERNUMERARY TEETH : A LITERATURE REVIEW

Miguel E. León, Melissa Mejía

33 CLASIFICACIÓN DE LOS DEFECTOS DE EXTENSIÓN DE DIENTES ANTERIORES TRATADOS ENDODÓNTICAMENTE

CLASSIFICATION PROPOSAL TO MEASURE EXTENSION DEFECTS OF ENDODONTICALLY TREATED ANTERIOR TEETH

Rafael Murgueitio

38 MANEJO DE LA VÍA ORAL EN TRAUMA MAXILOFACIAL

MANAGEMENT OF AIRWAY IN MAXILLOFACIAL

Juán S. Martínez, Carolina Valencia 


\section{Revista Estomatología}

\section{Contribuciones}

La Revista Estomatología tiene especial interés en publicar colaboraciones originales inéditas, que abarquen las ciencias naturales y humanas, la biotecnología y la cultura, incluídas las bellas artes, sin que tales intereses alteren la amplitud con que se ofrece esta tribuna a la gente pensante de Colombia y el mundo, pero de manera especial a los estudiantes de los niveles "Auxiliar", "Técnico Simple", "Técnico Profesional", "Tecnológico" y "Profesional" de pregrado y postgrado, a los profesores de la Escuela, a los de las otras Escuelas de la Facultad de Salud incluida la Escuela de Salud Pública y sus centros especiales (CEDETES, CISALVA, etc.) así como a estudiantes y profesores del Instituto de Psicología, de la Facultad de Comuncación Social y, en general, a los investigadores en odontoestomatología.

La Revista Estomatología pretende continuar siendo una tribuna de divulgación científica, dedicada a difundir con un estiloágil y atractivo, así como con enfoques adecuados al lector no especializado, conceptos, ideas, métodos y resultados de investigaciones. Pero también avances sobre estudios en ejecución, sin detallar a profundidad el desarrollo de investigaciones en curso.

La Revista Estomatología incorpora contribuciones de naturaleza distina así:

Editoriales. Documentos que reflejan la posición del Consejo Editorial de la Revista frente a un determinado problema o tópico. Ordinariamente son producidos por el Director o por el Editor Asociado de la Revista Estomatología pero, también pueden ser elaborados por personas diferentes a los miembros del Consejo, a petición expresa del mismo. Para esta sección no se aceptan contribuciones espontáneas.

Cartas al Editor. Comunicaciones breves que busquen aclarar o complementar conceptos originados en los contenidos de los artículos publicados en la revista Estomatología.

Trabajos de Investigación y Artículos Originales. Incluye los informes finales de los trabajos de indagación científica que pueden ser de interés para los lectores de la Revista. las contribuciones para esta sección se acogen a las que rigen la literatura odontológica científica mundial. Los detalles aparecen en las Normas de Publicación.
De la Literatura Odontológica. Se aceptan contribuciones de excepcional interés que hayan sido publicadas en otras revistas del exterior. En este caso, siempre se deberá mencionar si se ha publicado previamente y se deberán incluir la referencia original y el permiso del correspondiente editor.

De la Actualización en Salud Oral. Se pueden presentar revisiones completas y exhaustivas sobre diferentes tópicos, cuyo propósito sea poner al día a los lectores respecto a los avances en el campo de la ciencia. Los contribuyentes serán personas de reconocido prestigio en el campo de su práctica y, ordinariamente, deben incluir abundantes referencias recientes.

Comunicaciones Breves. Notas cortas de máximo 2 páginas tamaño carta escritas a doble espacio, en las cuales se trata de expresar una enseñanza sobre un tema específico o comentar un problema reciente o plantear una inquietud. Su bibliografía se limita a 10 referencias.

Contribuciones de Artes y Cultura. Se aceptan artículos de interés en relación con la literatura (narrativa, poesía, ensayos filosóficos) o con las manifestaciones de las artes plásticas. la condición es que tengan los requerimientos de las comunicaciones breves.

Los artículos publicados en la Revista Estomatología son de responsabilidad exclusiva del Autor (o Autores) y no reflejan necesariamente el criterio o el pensamiento del Consejo Editorial.

Dirigir todos los manuscritos y correspondencia a:

\section{Revista Estomatología}

odontologia@univalle.edu.co

Dr. José Fernando Barreto, Editor Asociado

Escuela de Odontología, Facultad de Salud

Universidad del Valle, Sede San Fernando

Calle $3 A N^{\circ} 36 B-00$, Edificio 132, Oficina 307

Apartado Aéreo 25360

Cali, Colombia 


\section{Editorial}

L

uego de 16 años de existencia de la Revista Estomatología de la Universidad del Valle, se puede manifestar que ésta se encuentra consolidada como el órgano de difusión del nuevo conocimiento generado a partir de la investigación, de la experiencia clínica y de la lectura crítica de la literatura, no sólo de los docentes y estudiantes de pre y postgrado de la Escuela de Odontología de la Universidad del Valle, sino también de los profesionales de la salud buco-maxilo-facial del suroccidente colombiano. Es así como al hacer un balance, en los 15 volúmenes (25 números) realizados se han logrado publicar un total de 187 artículos, de los cuales el 36.8\% corresponde a artículos originales, el 14\% a reportes de casos y el $50.2 \%$ a revisiones de la literatura.

A la fecha, dentro de los objetivos primordiales del consejo editorial se encuentran lograr la indexación en la Base Bibliográfica Nacional Publindex de COLCIENCIAS, fortalecer el comité científico internacional, continuar con el desarrollo de la versión on-line de la revista bajo la plataforma electrónica Open Journal System e incentivar la recepción de artículos mediante la publicación de suplementos que cubran temas específicos inherentes a la salud estomatológica.

Por tanto, el reto implica el trabajo ininterrumpido del consejo editorial con la decidida colaboración de la dirección de la Escuela de Odontología de la Universidad del Valle y del Programa Editorial de la Vice-rectoría de Investigaciones de la misma universidad.

Freddy Moreno Gómez Editor Invitado.

Santiago de Cali, Diciembre de 2.007 